\title{
Ultrafast Resonant Interatomic Coulombic Decay Induced by Quantum Fluid Dynamics
}

A. C. LaForge ${ }_{1}^{1,2,{ }^{*}}$ R. Michiels, ${ }^{2}$ Y. Ovcharenko $\odot,{ }^{3,8}$ A. Ngai, ${ }^{2}$ J. M. Escartín $\odot,{ }^{4}$ N. Berrah $\odot,{ }^{1}$ C. Callegari®$\odot,{ }^{5}$ A. Clark, ${ }^{6}$ M. Coreno, ${ }^{7}$ R. Cucini, ${ }^{5}$ M. Di Fraia,${ }^{5}$ M. Drabbels, ${ }^{6}$ E. Fasshauer, ${ }^{8}$ P. Finetti, ${ }^{5}$ L. Giannessi, ${ }^{5,9}$ C. Grazioliø ${ }^{18}{ }^{18}$ D. Iablonskyi ${ }^{10}$ B. Langbehn $\oplus,{ }^{3}$ T. Nishiyama, ${ }^{11}$ V. Oliver, ${ }^{6}$ P. Piseri, ${ }^{12}$ O. Plekan, ${ }^{5}$ K. C. Prince $\odot,{ }^{5}$ D. Rupp, ${ }^{3,9}$ S. Stranges $\odot,{ }^{13}$ K. Ueda,${ }^{10}$ N. Sisourat, ${ }^{14}$ J. Eloranta, ${ }^{15}$ M. Pi, ${ }^{16,17}$ M. Barranco,${ }^{16,17}$ F. Stienkemeier $\odot,{ }^{2}$

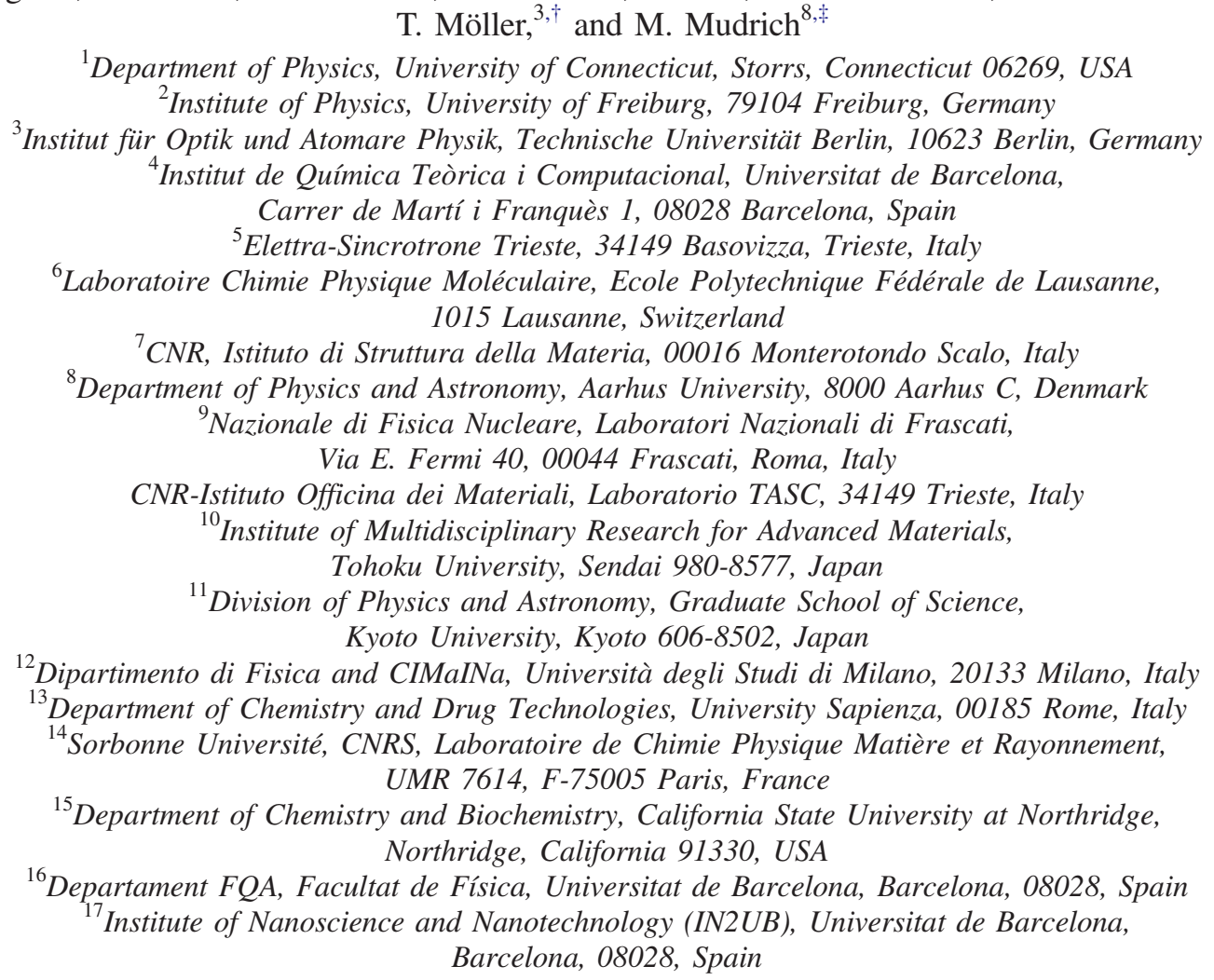

(Received 3 September 2020; revised 5 January 2021; accepted 19 February 2021; published 12 April 2021)

\begin{abstract}
Interatomic processes play a crucial role in weakly bound complexes exposed to ionizing radiation; therefore, gaining a thorough understanding of their efficiency is of fundamental importance. Here, we directly measure the timescale of interatomic Coulombic decay (ICD) in resonantly excited helium nanodroplets using a high-resolution, tunable, extreme ultraviolet free-electron laser. Over an extensive range of droplet sizes and laser intensities, we discover the decay to be surprisingly fast, with decay times as short as $400 \mathrm{fs}$, nearly independent of the density of the excited states. Using a combination of timedependent density functional theory and $a b$ initio quantum chemistry calculations, we elucidate the mechanisms of this ultrafast decay process, where pairs of excited helium atoms in one droplet strongly attract each other and form merging void bubbles, which drastically accelerates ICD.
\end{abstract}

DOI: 10.1103/PhysRevX.11.021011

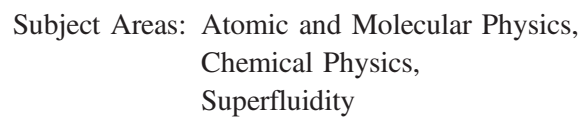

*aaron.laforge@uconn.edu

†thomas.moeller@physik.tu-berlin.de

*mudrich@phys.au.dk

${ }^{\S}$ Present address: European XFEL, 22869 Schenefeld, Germany.

"Present address: Laboratory for Solid State Physics, ETH Zurich, 8093 Zurich, Switzerland.

Published by the American Physical Society under the terms of the Creative Commons Attribution 4.0 International license. Further distribution of this work must maintain attribution to the author(s) and the published article's title, journal citation, and DOI. 


\section{INTRODUCTION}

In molecular and condensed-phase systems, interatomic processes offer a means for localized energy or charge to disperse to the surrounding environment. The diversity of these processes often makes it tedious or impossible to obtain a complete picture of the ionization mechanisms and dynamics. Alternatively, free, weakly bound nanosystems, such as van der Waals (vdW) clusters, can be used to study such interatomic interactions in a well-controlled manner. In particular, the study of vdW clusters irradiated by intense extreme ultraviolet (XUV) free-electron laser (FEL) radiation has led to the observation of numerous novel interatomic processes [1-3]. An important type of interatomic process known as interatomic Coulombic decay (ICD) [4] has been widely studied in weakly bound systems [5-7]. In cases where local Auger decay is energetically forbidden, an excited atom or molecule releases its excitation energy by transferring it to a neighboring atom or molecule, which can result in its ionization. In general, ICD is a prominent decay mechanism in a multitude of systems, specifically those of biological relevance [8-11]. A key parameter in determining the importance of ICD in a nanosystem is its decay time, which is directly linked to its efficiency. With the advent of seeded FELs and the availability of intense, tunable XUV radiation [12,13], new types of resonant ICD [14] have been observed in vdW clusters [15-18], where at least two photons are absorbed and energy is exchanged between neighboring excited atoms. More importantly, FELs offer the capability to directly follow the dynamics of ICD in the time domain. So far, ultrafast time-resolved measurements have been limited to ICD in vdW dimers [19,20]. Although vdW dimers, a type of weakly bound molecular system, are of fundamental interest, they can by no means exemplify the complex dynamics occurring in a nanoparticle or condensed-phase system where multiple intermolecular processes can play a critical role.

He nanodroplets serve as model nanosystems in numerous research fields, since they can bridge the gap between simple atomic and molecular systems and extended, condensedphase systems [21]. Because of their extremely weak interatomic binding, superfluid nature, and simple electronic structure, He nanodroplets are an ideal environment to study interatomic processes in a homogeneous liquid $[15,16,18,22,23]$ or in a well-defined heterocluster, as is the case for doped He nanodroplets [24-29]. When the He environment is excited, a void bubble, or "cavity," is formed around the excited atom [30,31], which can freely move about the droplet. This phenomenon is a general feature in closed-shell systems where the local environment tends to exert a repulsive force on electrons or impurities with a low electron affinity due to the Pauli exclusion principle. This interaction induces the formation of cavities around the impurities and possible clustering or assembly thereof [32]. A similar, although more complicated, process of cavity formation occurs in classical fluids and is responsible for

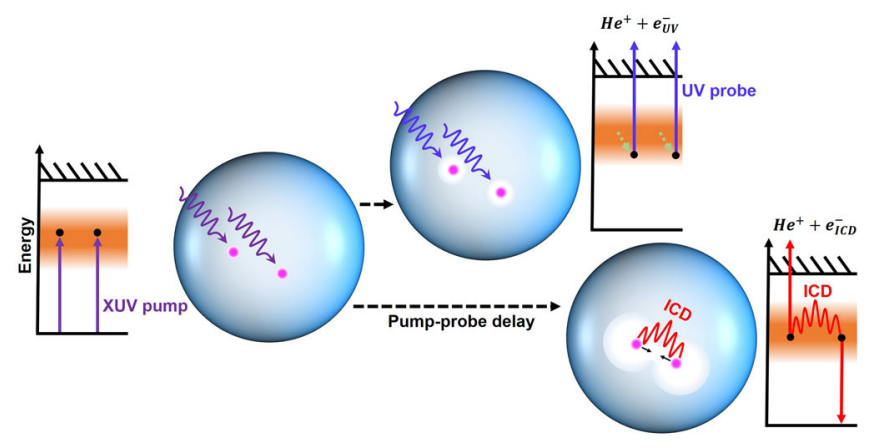

FIG. 1. Atoms in the He droplet are excited to the $1 s 2 p$ droplet band by the XUV pump pulse. After internal relaxation, bubbles are formed around the excited atoms. The two bubbles are accelerated toward each other, leading to a merging of the bubbles and ICD (bottom scenario). A time-delayed UV pulse is used to probe the dynamics of the process. If the UV probe pulse comes before ICD occurs, the pulse ionizes the excited atoms, thereby halting the ICD process (top scenario at short pump-probe delay). The He droplet is represented by the large blue circles, and excited atoms and bubbles are represented by pink and white circles, respectively. The energy-level diagrams illustrate the energetics of processes. Using photoelectron spectroscopy, one can differentiate ICD electrons and UV-ionized electrons as shown in Fig. 2.

molecular solvation. For example, in water, the so-called hydrophobic effect determines whether a molecule is individually hydrated or assembles into a larger structure [33].

Here, we report on time-resolved measurements of resonant ICD in nanoparticles, where $\mathrm{He}$ droplets are chosen as a model system. The process is schematically shown in Fig. 1. Atoms are excited by an XUV pulse tuned into resonance with the $1 s 2 p$ droplet band $(h \nu=21.6 \mathrm{eV})$ [34]. The pulse creates multiple excited atoms in the droplet which can decay via ICD; i.e., the energy from one excited $\mathrm{He}$ atom, $\mathrm{He}^{*}$, is transferred to another $\mathrm{He}^{*}$, which, in turn, is ionized. A second, time-delayed UV pulse can directly ionize the excited atom(s) in the droplet, thereby interrupting and halting any interatomic decay processes. Over an extensive range of droplet sizes and laser pulse energies, the decay mechanism is found to be much faster than predicted by theory [14]. Even more surprisingly, the decay rate is nearly independent of the number of excited atoms per droplet, although theory predicts a very strong dependence on the internuclear distance [14]. To understand these discrepancies, the experimental results are modeled using a combination of time-dependent density-functional theory (TDDFT) [35,36] and ab initio calculations of the doubly excited $\mathrm{He}^{*}-\mathrm{He}^{*}$ pair potentials as well as the ICD widths.

We discover that the ICD dynamics are largely determined by the attractive interaction of closely spaced $\mathrm{He}^{*}$ atoms and by the formation of bubbles around them. The latter strongly accelerates the ICD via the merging of overlapping bubbles. Previous experimental work on bubble formation in He droplets [31] focuses on the relaxation 
of one excitation in a droplet, which forms a single bubble in the course of its relaxation. In contrast, here, the focus is on ultrafast dynamics between two bubbles, which facilitates the ICD process. Overall, the relevance of our findings is (i) realizing that, in a condensed-phase system, ICD is not only determined by the electronic interaction of the two atoms involved, but it can be crucially influenced by the response of the surrounding medium, and (ii) the resulting ICD time is drastically reduced to the femtosecond range, in contrast with previous expectations that this type of ICD is much slower, i.e., tens to hundreds of picoseconds $[14,17]$.

\section{EXPERIMENT}

This work is performed at the low density matter end station [37] of the seeded FEL FERMI, in Trieste, Italy. The FEL photon energy $(21.6 \mathrm{eV})$ is tuned via the seed laser, undulator gaps, and other machine parameters, yielding a pulse length of approximately $100 \mathrm{fs}$ full width at half maximum (FWHM) $[12,13]$. The measurements are taken over two separate periods with different experimental conditions. The second set of parameters is given in parentheses. The FEL pulse energy, varied from 0.1 to $50 \mu \mathrm{J}$, is determined upstream by gas ionization, taking the nominal reflectivity of the optical elements in the beam transport system into account. The diameter of the FEL focus is $250 \mu \mathrm{m}$ FWHM. The UV probe pulse is obtained from a frequency-tripled (-doubled) Ti:sapphire laser $\left[h \nu^{\prime}=4.8(3.2) \mathrm{eV}\right]$ with a pulse energy of $50(200) \mu \mathrm{J}$ and a focus diameter of $250 \mu \mathrm{m}$ FWHM. A tin filter of $160 \mathrm{~nm}$ thickness is used to suppress higher-order harmonic radiation. The cross-correlation between the FEL and the probe laser is $200 \mathrm{fs}$ FWHM, measured by resonant two-photon ionization of $\mathrm{He}$ gas. A supersonic jet of $\mathrm{He}$ nanodroplets is produced by expansion of high-pressure $\mathrm{He}$ gas through a pulsed, cryogenically cooled Even-Lavie nozzle. By varying the expansion conditions (backing pressure and nozzle temperature), the mean cluster size is varied in the range of $\langle N\rangle=10^{2}-10^{5} \mathrm{He}$ atoms [38]. The nanodroplet beam is perpendicularly crossed by the FEL and UV beams at the center of a velocity map imaging spectrometer [37]. The electron kinetic energy distributions are reconstructed using the maximum entropy velocity Legendre reconstruction method [39].

\section{RESULTS AND DISCUSSION}

Figure 2(a) shows the distributions of electron kinetic energy $E_{e}$ emitted by resonantly excited He droplets as a function of the delay between XUV pump and UV probe laser pulses. The mean droplet size is $\langle N\rangle=76000$ atoms, and the XUV intensity is $2.8 \times 10^{9} \mathrm{~W} / \mathrm{cm}^{2}$. At low kinetic energies $\left(0<E_{e}<2 \mathrm{eV}\right)$, the electron distribution is created by resonant two-photon ionization (2PI) in $\mathrm{He}$ nanodroplets. At short delays, $\Delta t<1 \mathrm{ps}$, the distribution shows the droplet-induced relaxation dynamics of $\mathrm{He}^{*}$ from the XUV-excited $1 s 2 p$ state to the $1 s 2 s$ state [31]. At higher kinetic energies $\left(15<E_{e}<18 \mathrm{eV}\right)$, resonant multiphoton ICD is observed according to the reaction [14-16,18]

$$
\left(\mathrm{He}^{*}+\mathrm{He}^{*}\right) \mathrm{He}_{N-2} \rightarrow\left(\mathrm{He}^{+}+e_{\mathrm{ICD}}+\mathrm{He}\right) \mathrm{He}_{N-2} .
$$

Here, $\mathrm{He}_{N-2}$ denotes the He droplet, and $e_{\mathrm{ICD}}$ is the ICD electron. A discussion of the electronic states which initiate this type of ICD is given in the Appendix A. Since resonant ICD is a binary process, at least two excited atoms are required per droplet.

The intensities of photoelectrons (red squares) and ICD electrons (blue circles), depicted in Fig. 2(b), display opposing trends in their time evolution: The 2PI signal
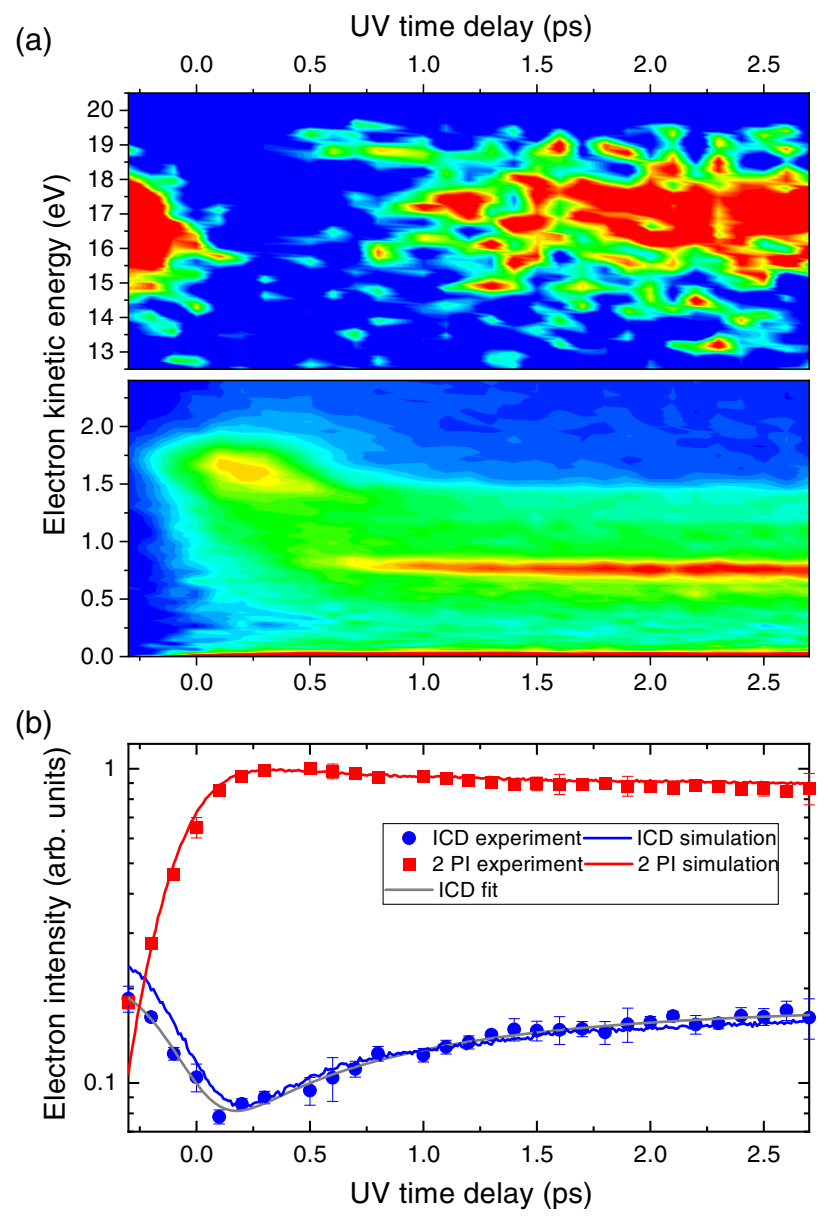

FIG. 2. (a) Time-resolved electron kinetic energy distributions of resonantly excited He droplets centered around the ICD peak (top) and the two-photon ionization (2PI) signal (bottom). (b) Projection of the intensity of the ICD peak (blue circles) and the 2PI signal (red squares) as a function of XUV-UV pumpprobe delay. The experimental data are fitted with an exponential decay function convoluted with a cross correlation function (gray line). The red and blue lines show the results of a MC simulation (see the text for details). The droplet size is 76000 atoms, and the excitation photon energy is $21.6 \mathrm{eV}$. 


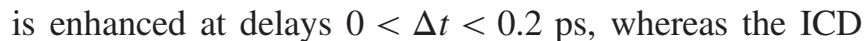
signal is depressed. This result can be rationalized by the depletion of the $\mathrm{He}^{*}$ population through photoionization by the UV probe pulse, thereby suppressing the ICD. As the pump-probe delay is increased, ICD can proceed before the $\mathrm{He}^{*}$ are photoionized and the $e_{\mathrm{ICD}}$ yield is replenished. Thus, the rise of the $e_{\mathrm{ICD}}$ yield reflects the timescale of the ICD. To quantify this process, the ICD signal is fitted with a function [gray line in Fig. 2(b)] accounting for the exponential rise as well as the pump-probe cross correlation. A thorough discussion of the fitting procedure is given in Appendix B. Figure 2(b) also shows the ICD (blue line) and 2PI (red line) data from a Monte Carlo (MC) simulation as discussed later in the text. Additional data and fits for different experimental parameters are given in Appendix C.

To systematically investigate the dynamics of ICD in $\mathrm{He}$ nanodroplets, pump-probe delay dependences over a wide range of He droplet sizes and XUV intensities are recorded. The latter controls the $\mathrm{He}^{*}$ excitation probability (photon flux $\times$ absorption cross section) and thereby the mean distance between $\mathrm{He}^{*}$ in a droplet. Because of the strong coupling between the FEL power, droplet size, and collective autoionization (CAI) effects [18], only a limited range of excitation probabilities $(0.1 \%-1 \%)$ show a clearly distinguishable ICD peak, despite the broad range of droplet sizes and FEL intensities available. As the FEL intensity increases, multiple excited atoms may interact, leading to decay by CAI and formation of a nanoplasma [16]. In the transition from ICD to CAI, the ICD peak broadens and shifts to lower energies due to the formation of a collective Coulomb potential and eventually becomes dominated by low-energy thermal electrons from the nanoplasma [18].

Similar to what is shown in Fig. 2(b), each ICD delay dependence is fitted with a function to determine the time constant of the $e_{\mathrm{ICD}}$ evolution. The resulting ICD times $\tau_{\mathrm{ICD}}$ and $e_{\mathrm{ICD}}$ yields are, respectively, plotted as red symbols in Figs. 3(a) and 3(b) as a function of the excitation probability. The corresponding MC simulation results are shown as blue dots. The $e_{\mathrm{ICD}}$ yield is determined from the total number of detected electrons and the $\mathrm{He}^{*}$ photoionization cross section [40]. It is normalized to the number of $\mathrm{He}^{*}$ atoms in the droplet and multiplied by 2 to account for the fact that two excitations produce one ICD electron. The resulting ICD efficiency rises from 0.09 to 0.32 in the given range of $\mathrm{He}^{*}$ excitation probability, while $\tau_{\mathrm{ICD}}$ decreases from 1000 to $400 \mathrm{fs}$. To decouple the effect of the droplet size from the FEL intensity, we additionally perform MC simulations (see Supplemental Material [41] for details) for fixed droplet sizes. The results for small and large droplets are shown in Fig. 3 as black and gray lines, respectively. For small droplets, the ICD time is nearly constant and lower than the ICD decay times for large droplets, which show a weak dependence on the FEL intensity. The ICD efficiency shown in Fig. 3(b) rises from zero as a function

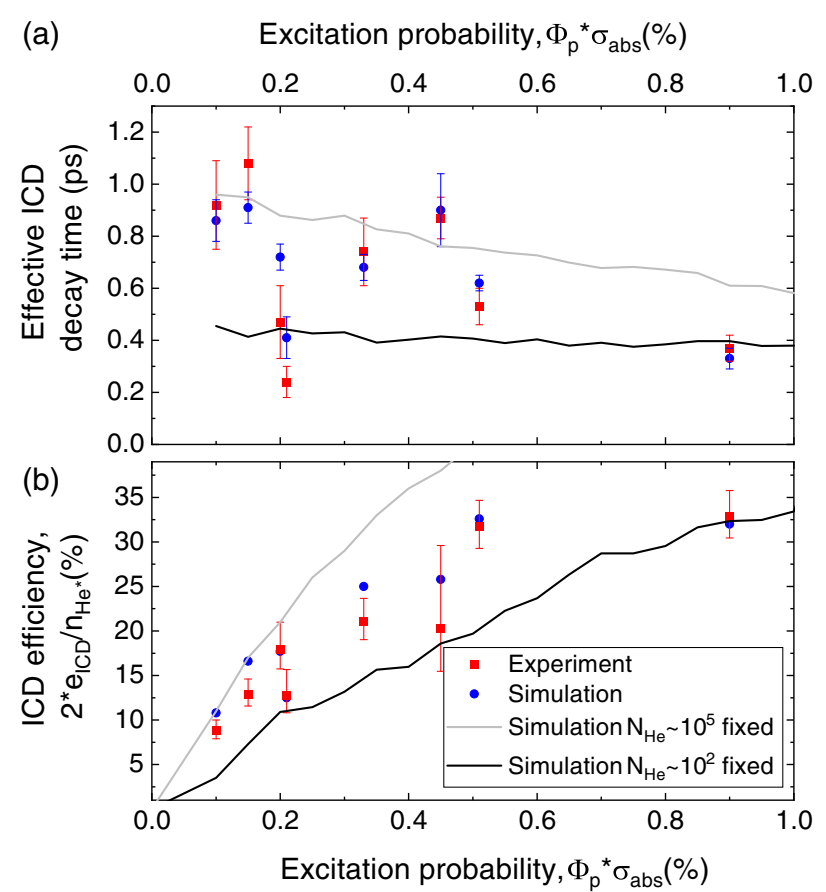

FIG. 3. (a) Effective ICD decay time and (b) ICD efficiency plotted as a function of the excitation probability (red squares). MC simulation results for the specific experimental conditions, droplet size, and FEL intensity are shown as blue dots. Additionally, to show the general trend in the simulations on the FEL intensity, the results for fixed small and large droplets are shown as black and gray lines, respectively.

of the excitation probability with a higher slope for large droplets. Overall, the MC simulations are in excellent agreement with the experimental data.

In general, the measured ICD decay times are surprisingly short $\left(\tau_{\mathrm{ICD}}<1 \mathrm{ps}\right)$ compared to estimates based on the virtual photon ICD model for this type of decay, which yield 52 ps for the fastest channel [14]. Furthermore, previous static measurements predict this type of ICD to be much slower, in the high picosecond range [17,28]. Further proof of the discrepancy between theory and experimental results can be seen in Fig. 4(c), which shows the ICD decay width $\Gamma$ as a function of the $\mathrm{He}^{*}-\mathrm{He}^{*}$ distance. $\Gamma(d)$ is calculated by the Fano-CI-Stieltjes method [42] for all possible combinations of electronic states populated during droplet relaxation [28,31] (see Supplemental Material [41] for details). $\Gamma$, which is inversely proportional to the decay time $\tau_{\mathrm{ICD}}$, shows a very strong dependence on the $\mathrm{He}^{*}-\mathrm{He}^{*}$ distance. On the other hand, the measured $\tau_{\mathrm{ICD}}$, in Fig. 3(a), shows only a weak dependence on the $\mathrm{He}^{*}$ excitation probability, which is a measurable quantity proportional to the mean $\mathrm{He}^{*}-\mathrm{He}^{*}$ distance. The observed ultrafast ICD rates, in the femtosecond regime, can be explained only through an additional mechanism that brings the two $\mathrm{He}^{*}$ atoms into close contact. Excitation migration [43,44], excitation 
delocalization [45], and hole hopping have been discussed extensively over the years, especially in the context of Penning ionization $[44,46]$. While fast excitation transfer, akin to exciton hopping, can explain the high efficiency of the Penning process [44], it cannot account for the short ICD lifetime. Delocalization of excitations over an extended region of the $\mathrm{He}$ droplet as a consequence of exciton hopping would lead to a reduced local spatial overlap and, thus, to low ICD rates. Besides, the large variation of the interatomic distances between $\mathrm{He}$ atoms in the droplets due to the large zero-point motion as well as many-body quantum effects may also limit delocalization $[45,46]$. Unfortunately, the problem of excitation transfer in superfluid $\mathrm{He}$ has not yet been addressed theoretically, despite the numerous experimental Penning ionization studies. That said, an additional mechanism is required that brings two $\mathrm{He}^{*}$ into close contact such that ICD takes place at short distances.

Aside from the fast delay-time dependence of the ICD signal, we observe that the $e_{\mathrm{ICD}}$ yield in most cases does not fully rise to the level measured at negative delays within the full range of pump-probe delays; see Appendixes B and C. This result indicates that some of the $\mathrm{He}^{*}$ decay by ICD much more slowly than the experimentally observed asymptotic value from which we deduce $\tau_{\text {ICD }}$. Furthermore, the observation that the ICD efficiency never exceeds $35 \%$ in our experiments points at a competing

(a)
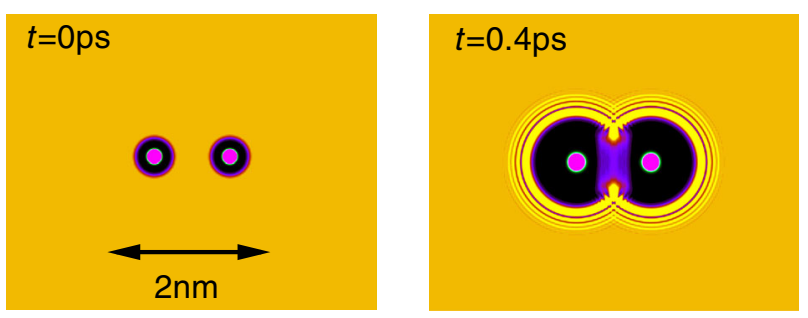

(b)

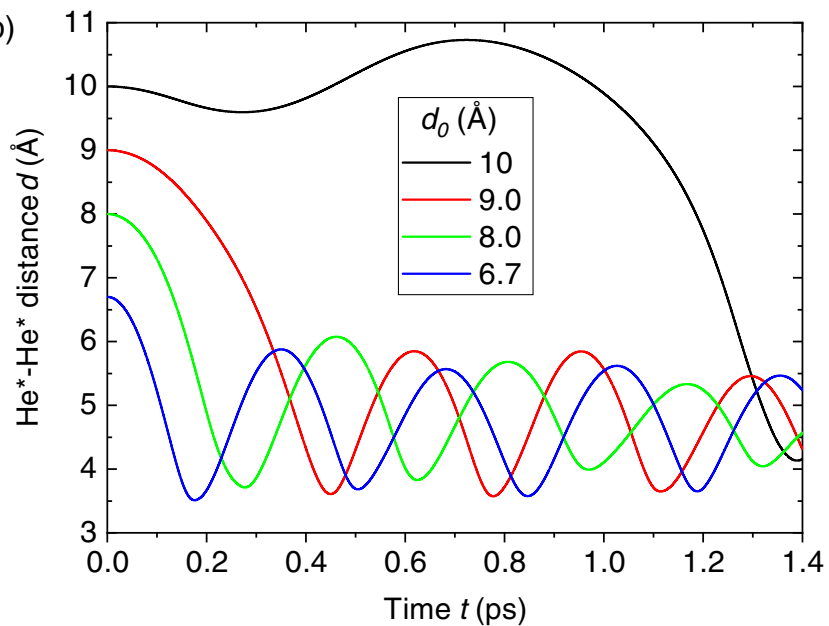

relaxation channel that prevents the majority of $\mathrm{He}^{*}$ from decaying via ICD.

To better understand the response of He nanodroplets to multiple excitations and to rationalize our experimental findings, TDDFT simulations are performed [31,35,36,47] for the motion of $\mathrm{He}^{*}$ pairs. To keep the simulations tractable, we consider bulk superfluid $\mathrm{He}$, which is coupled to the $\mathrm{He}^{*}$ pair self-consistently. Because of the light mass of the $\mathrm{He}^{*}$ "impurities," they must be treated quantum mechanically with the potential term given by the $\mathrm{He}^{*}$-droplet interaction. To include the interaction between the two $\mathrm{He}^{*}$ atoms, the $\mathrm{He}^{*}-\mathrm{He}^{*}$ pair potentials are calculated using highly correlated $a b$ initio methods (see Supplemental Material [41] for details).

Figure 4(a) shows the time evolution of the 2D cuts of the He density distribution (yellow-red area) when the two excited $\mathrm{He}$ atoms are initially separated by $d_{0}=10 \AA$ (pink-green dots). Animations of these simulated dynamics for various initial conditions are included in Supplemental Material [41]. Upon excitation, bubbles form around them due to the repulsion between the Rydberg electrons and the surrounding closed-shell $\mathrm{He}$ atoms [30,31,47-49]. As the bubbles grow and the two $\mathrm{He}^{*}$ atoms weakly attract each other, the bubbles eventually overlap and merge into one large bubble. The salient feature is that, shortly after the two bubbles coalesce, the two $\mathrm{He}^{*}$ are strongly accelerated toward each other. This process is facilitated by the merging
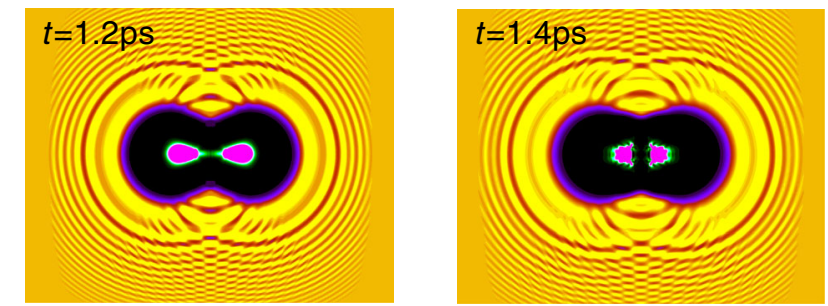

(c)

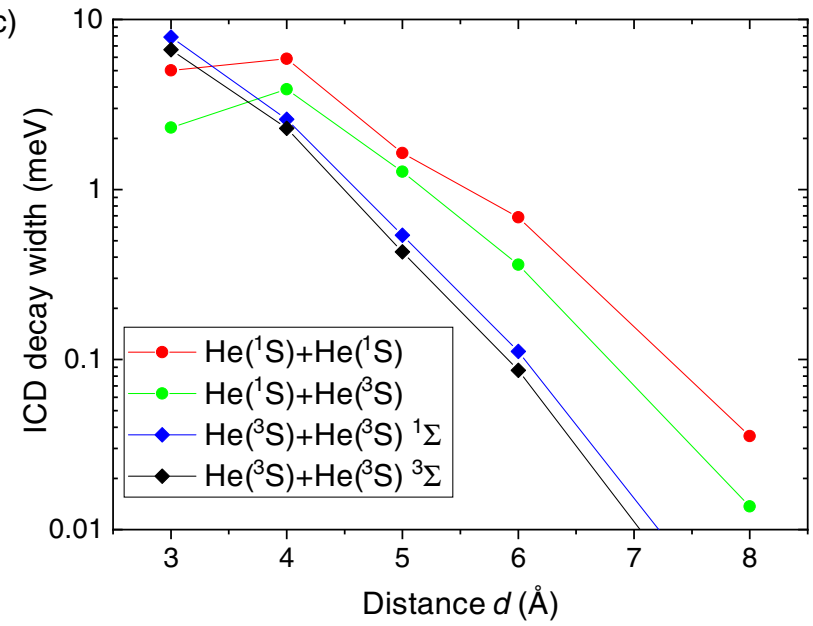

FIG. 4. (a) Snapshots of the He density evolution around two He* centers separated initially by $d_{0}=10 \AA$. The probability distribution of $\mathrm{He}^{*}$ is represented as pink dots, and the black areas show the void bubbles forming. (b) Evolution of the He*-He* distance $d$ for various values of the initial distance $d_{0}$. (c) Calculated ICD widths for various combinations of $1 s 2 s$-excited He* atomic states. 
of the bubbles where the $\mathrm{He}^{*}$ reach interatomic distances $d<4 \AA$ within 400 fs for all initial distances $d_{0}$ up to $9 \AA$; see Fig. 4(b). As ICD is not explicitly included in the TDDFT simulations, the $\mathrm{He}^{*}$ pair continues vibrating at a short distance due to the attractive $\mathrm{He}^{*}-\mathrm{He}^{*}$ potential. However, within the first half cycle of the vibration, the ICD decay width reaches $\Gamma(d=4 \AA)=5.9 \mathrm{meV}$, corresponding to a characteristic ICD time $\tau_{\mathrm{ICD}}^{\text {theo }}=110$ fs for the $\operatorname{He}^{*}\left({ }^{1} S\right)+$ $\mathrm{He}^{*}\left({ }^{1} S\right)$ pair, which has the largest branching ratio in the droplet relaxation $[28,31]$. Thus, all $\mathrm{He}^{*}$ pairs with $d_{0} \lesssim 10 \AA$ actually decay via ICD within $t \lesssim 1.4$ ps with a probability of near unity. Thus, we conclude that the decay in this particular system is largely determined by the quantum fluid dynamics of the merging bubbles, as well as the pairwise attraction of excited atoms.

For larger initial distances $\left(d_{0}>10 \AA\right)$, the time between $\mathrm{He}^{*}$ excitation and bubble merging quickly increases to $t>10 \mathrm{ps}$, and, therefore, ICD becomes very slow. This result explains the observed incomplete replenishment of the $e_{\mathrm{ICD}}$ signal at long pump-probe delays. The question remains why not all $\mathrm{He}^{*}$ decay by ICD, in the absence of the probe pulse. It is known that radiative decay is not expected to play a significant role, since the lifetime is in the nanosecond range [30,50]. Previous experimental and theoretical studies show that, following the bubble formation, some of the $\mathrm{He}^{*}$ remain weakly bound to the $\mathrm{He}$ droplet surface, where they eventually form $\mathrm{He}_{2}^{*}$ excimers [51], whereas others are directly ejected from the droplets $[31,48,52]$. Once a $\mathrm{He}^{*}$ detaches from the droplet, it can no longer decay via ICD, but it still contributes to the photoionization signal. Based on our measurements [Fig. 3(b)], the fraction of ejected $\mathrm{He}^{*}$ is estimated to be larger than $50 \%$. The competition between direct ejection and ICD for initial distances $d_{0}>10 \AA$ is strongly dependent on the droplet size.

The ICD dynamics in $\mathrm{He}$ nanodroplets are largely governed by the motion of $\mathrm{He}^{*}$ driven by the bubble dynamics and the interatomic $\mathrm{He}^{*}-\mathrm{He}^{*}$ potential, competing against the ejection of surface $\mathrm{He}^{*}$ from the droplet. To account for the aforementioned effects, a simplified MC simulation based on $\Gamma(d)$ is developed. The results are displayed in Fig. 3(b). The He droplet is treated as a homogeneously packed sphere of He atoms represented by equally sized spheres. An initial number of $\mathrm{He}^{*}$, according to the XUV intensity and the He droplet absorption cross section [34], are placed at random positions within the droplet. Then, for each $\mathrm{He}^{*}$, the following conditions are tested. If the distance to the droplet surface $d_{S}<7.5 \AA$ and the distance to the nearest-neighbor $\mathrm{He}^{*} d_{0}>9.5 \AA$, then the $\mathrm{He}^{*}$ is ejected. If $d_{S}>7.5 \AA$ and $d_{0}<15.5 \AA$, then the $\mathrm{He}^{*}$ undergoes ICD; the ICD probability is then calculated based on $\Gamma(d)$ according to the trajectory $d(t)$ obtained from the TDDFT simulations. If $d_{S}>7.5 \AA$ and $d_{0}>15.5 \AA$, the $\mathrm{He}^{*}$ does not decay by ICD and only photoionization is possible. The probe pulse is implemented by converting the $\mathrm{He}^{*}$ into photoelectrons at a rate consistent with the experimental estimate. The values $d_{0}=$ $15.5 \AA$ and $d_{S}=7.5 \AA$, used as criteria for ICD enhanced by bubble merging and $\mathrm{He}^{*}$ ejection, respectively, are deduced from the TDDFT simulations. Additionally, when the same simulation is performed for fixed positions of $\mathrm{He}^{*}$, the ICD time constants are 1-2 orders of magnitude longer than the experimental values, thus demonstrating the importance of ultrafast bubble dynamics and the attractive $\mathrm{He}^{*}-\mathrm{He}^{*}$ potential. An in-depth discussion of these simulations is given in Appendix D.

Overall, the conceptual leap presented in our results show that resonant ICD in a superfluid nanosystem is predominantly driven by the ultrafast dynamics of the medium surrounding the interaction centers $\left(\mathrm{He}^{*}+\mathrm{He}^{*}\right.$, in this case). Previous work on (nonresonant) ICD considers only the dynamics of the interaction centers, not their environment. While this assumption is valid for small systems like dimers and crystalline clusters, in extended condensed-phase systems such as liquids and superfluids, the response of the local environment has to be taken into account. Our work directly demonstrates that the mesoscopic dynamics may drastically enhance the ICD rate. This result has implications for biological systems where the local aqueous medium surrounding the biomolecules may crucially impact the decay rate when being subjected to radiation damage by ICD $[10,11]$.

\section{CONCLUSIONS}

In summary, we have performed time-resolved measurements of resonant ICD in He nanodroplets. Over a wide range of droplet sizes and laser pulse energies, we have found the decay to be as fast as $400 \mathrm{fs}$ and to have little dependence on the density of excited states, in contrast to the strong dependence of the predicted ICD decay width on the distance between excitations. Our simulations have shown that the ICD dynamics are largely determined by the pairwise attraction of excited atoms, as well as the peculiar response of He droplets to multiple resonant excitations. The formation of bubbles around the excitations and their subsequent merging accelerates ICD, whereas the ejection of excited state atoms from the droplet competes with it. Thus, using He droplets as a model system bridging molecular and condensed-phase sciences, we have demonstrated that resonant ICD in the condensed phase is governed by ultrafast relaxation mechanisms that couple electronic and nanofluid translational degrees of freedom. These results pave the way for time-resolved measurements of more complex intermolecular decay processes involving He excited state dynamics in doped He droplets, such as ICD-mediated single or double ionization of molecules and clusters [26-28].

Although He nanodroplets are a unique type of quantum fluid, our findings of the bubble dynamics could have 
implications for other classical fluid systems where charge, excitations, or impurities can freely move throughout the medium. The dynamics of bubbles, also known as cavities in water, are responsible for a wide variety of fundamental processes, such as protein assembly into functional complexes in biological systems [33] or the behavior of impurities in metals, relevant to materials science [32]. Although classical fluids are more complex than He nanodroplets, intermolecular electronic decay and bubble dynamics are highly relevant processes in these systems. The range of intermolecular decay mechanisms extends beyond ICD [11] to processes such as electron transfer mediated decay [53], exciton-exciton annihilation [54], singlet fission [55], and Förster resonance energy transfer [56]. The rates of these decay mechanisms could be enhanced through bubble dynamics. In this regard, future time-resolved studies where the electronic decay and bubble dynamics could be controlled by laser excitation are of broad interest.

\section{ACKNOWLEDGMENTS}

The authors gratefully acknowledge financial support from the Carl-Zeiss-Stiftung, the Deutsche Forschungsgemeinschaft (DFG) under Grant No. MO 719/14-2, within the frame of the Priority Program 1840 "Quantum Dynamics in Tailored Intense Fields" (MU 2347/12-1 and STI 125/22-2), and the Carlsberg Foundation. TDDFT work has been performed under Grant No. FIS2017-87801-P (AEI/FEDER, UE) (M. B. and M. P.). A. C. L. and N. B. acknowledge the support of the Chemical Sciences, Geosciences and Biosciences Division, Office of Basic Energy Sciences, Office of Science, U.S. Department of Energy, Grant No. DESC0012376. D. R. acknowledges funding from Leibniz Grant No. SAW/2017/MBI4 and NCCR MUST of the Swiss National Science Foundation (SNF). J. E. acknowledges support from the National Science Foundation Grant No. DMR-1828019. J. M. E. acknowledges support from Ministerio de Ciencia e Innovación of Spain through the Unidades de Excelencia "María de Maeztu" Grant No. MDM-2017-0767.

\section{APPENDIX A: HIGH-RESOLUTION ICD ELECTRON KINETIC ENERGY DISTRIBUTION}

Information about the electronic states involved in the ICD process is encoded in the kinetic energy distribution of ICD electrons. Figure 5 shows a high-resolution electron spectrum measured at the photon energy $h \nu=23.7 \mathrm{eV}$. At this photon energy, the $1 s 4 p$ excited state of He droplets is resonantly excited [34]. The mean droplet size is set to $5 \times 10^{5} \mathrm{He}$ atoms. Besides the large signal component at low kinetic energy resulting from CAI [16], an additional peak is observed around $16 \mathrm{eV}$ with a shoulder near $15 \mathrm{eV}$, which is due to ICD. For comparison, we add vertical lines showing the expected ICD electron energies, $E_{e, \mathrm{ICD}}$, for

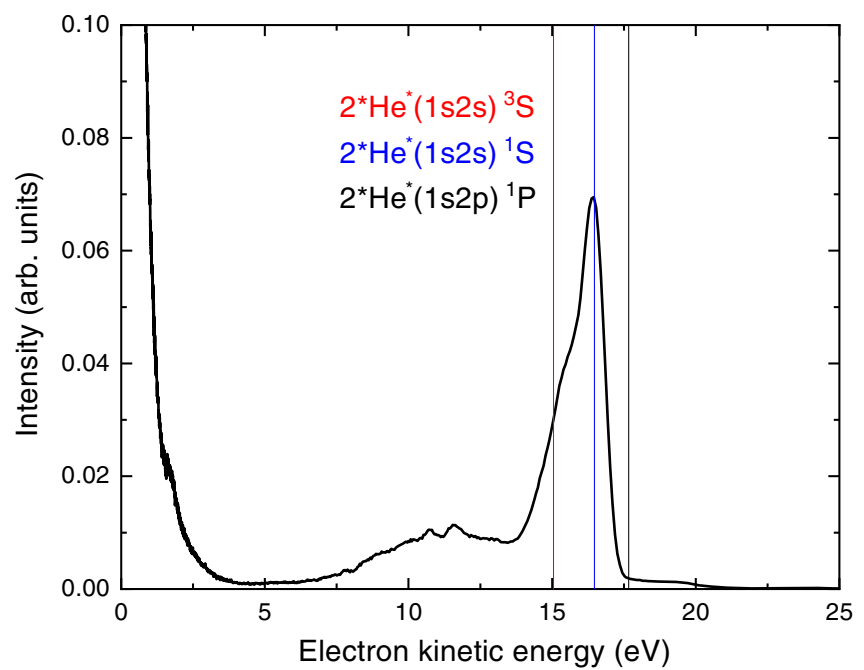

FIG. 5. Static (XUV only) electron kinetic energy distribution measured at $h \nu=23.7 \mathrm{eV}$. The vertical lines depict the nominal values of ICD electron energies for pairs of $\mathrm{He}^{*}$ atoms in the three lowest excited states.

pairs of $\mathrm{He}^{*}$ in the lowest excited states $2 s 2 s^{1,3} S$ and $2 s 2 p$ ${ }^{1} P$ according to

$$
E_{e, \mathrm{ICD}}=2 E_{\mathrm{He}(1 s 2 s, p)}-E_{i, \mathrm{He}} .
$$

Here, $E_{\mathrm{He}(1 s 2 s, p)}$ is the energy of the $1 s 2 s, p$ states of the He atom, and $E_{i, \mathrm{He}}$ is the He ionization potential. Clearly, the $1 s 2 s{ }^{1} S$ state is the dominant state producing ICD electrons. The $1 s 2 s{ }^{3} S$ state and $\mathrm{He}_{2}^{*}$ excimer states (broad feature around $E_{e, \mathrm{ICD}}=11 \mathrm{eV}$ ) also contribute but to a lesser extent. Although this electron spectrum is measured at a different excitation energy than those in the main text, ICD electrons appear to originate mostly from the same $\mathrm{He}^{*}$ states. This result is due to fast electronic relaxation, as previously observed in experiments using high-harmonic laser radiation [57], FEL [18,31], and synchrotron radiation [24,28].

\section{APPENDIX B: FITTING OF ICD ELECTRON YIELDS}

The time-dependent ICD electron intensities are fitted with a convolution of the pump-probe cross correlation function obtained by resonant two-photon ionization of $\mathrm{He}$ gas and an exponential decay leading to the following function:

$$
\begin{aligned}
I(t)= & I_{0}-A \operatorname{erfc}\left\{\left[\sigma^{2}-\tau\left(t-t_{0}\right)\right] /(\sqrt{2} \sigma \tau)\right\} \\
& \times \exp \left[-\left(t-t_{0}\right) / \tau\right]-B \operatorname{erfc}\left[\left(t-t_{0}\right) /(\sqrt{2} \sigma)\right] .
\end{aligned}
$$

This model is the simplest analytic function that reproduces the experimental measurements and is consistent with a more rigorous model of the pump-probe dynamics of ICD processes [58]. The exponential function describes the rise 


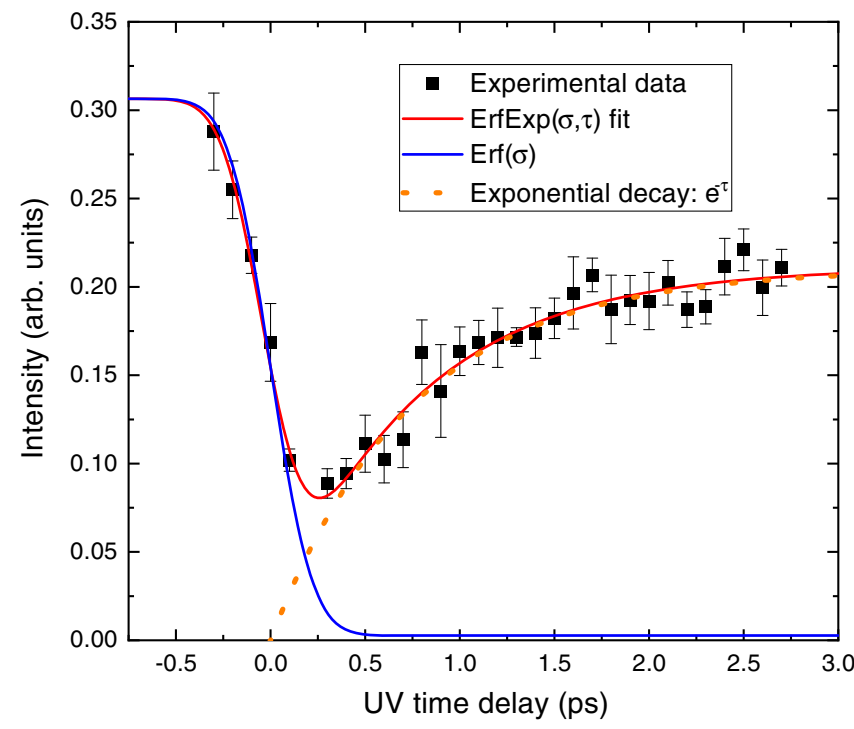

FIG. 6. Fitting of experimental time-resolved ICD data (black squares) with the function from Eq. (B1) (red curve). The components of the fit are illustrated separately: error function (blue curve) and exponential decay (orange dotted line).

of the electron counts for long delay times. Thus, the exponential decay constant $\tau$ represents the effective ICD time. The parameter $\sigma$ represents the cross-correlation width of the two overlapping laser pulses and is fixed to the value measured by resonant two-photon ionization of He gas. The time-zero value $t_{0}$ is constrained to $0 \pm 15 \mathrm{fs}$ in order to account for possible drifts in the FEL timing. The free parameters $I_{0}, A$, and $B$ reflect the total ICD intensity for $t \rightarrow-\infty, t \rightarrow \infty$, and the maximum depletion $I_{\min }$.

Figure 6 displays a fit of a typical experimental measurement. In addition to the full fit curve (red), we show the separate contributions from the error function (blue line) and exponential decay (orange dots).

\section{APPENDIX C: ADDITIONAL EXPERIMENTAL DATA}

To give a better overview of the experimental results and systematics, in Fig. 7(a), we show additional pumpprobe ICD electron yields measured under different experimental conditions. The red symbols correspond to small droplets with high excitation density. The resulting ICD curve is characterized by a fast time variation, as the mean interatomic distance between excited atoms is small, $d<10 \AA$, and, thus, ICD is fast. The black curve is for an intermediate excitation density and intermediate droplet sizes. The blue curve is for large droplets combined with a low excitation density. Replenishment of the ICD electron signal after depletion is slower, as ICD mostly occurs for pairs of $\mathrm{He}^{*}$ with larger initial separation. Figure 7(b) shows the results of the MC simulation for the same parameters as in the experiment. The good agreement

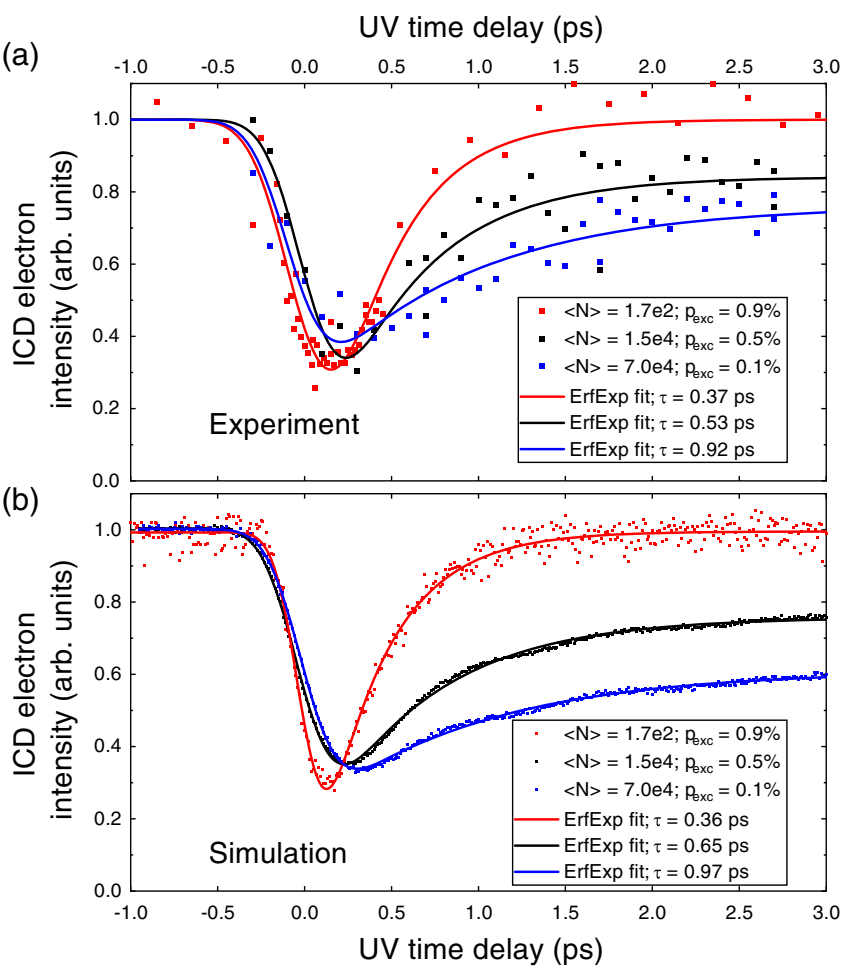

FIG. 7. (a) Experimental ICD electron intensities as a function of XUV-UV pump-probe delay with corresponding exponential fits for three different droplet sizes and excitation densities. (b) Simulated ICD electron intensities and fits for conditions similar to those of the upper panel.

shows that our model captures the main aspects of the pump-probe ICD dynamics.

\section{APPENDIX D: THE EFFECT OF ATOMIC MOBILITY ON ICD TIMESCALES}

Besides providing a deeper understanding of our experimental findings, MC simulations additionally allow us to ask more fundamental questions about the process, which cannot be directly addressed through experiment. For instance, how important is the mobility of the $\mathrm{He}^{*}$ atoms in the ICD process? To benchmark our simulations against a model system where the ICD rate is entirely given by the initial distances between $\mathrm{He}^{*}$, we carry out simulations where the $\mathrm{He}^{*}$ positions are held fixed. Figure 8(a) shows the simulated ICD electron intensity for stationary $\mathrm{He}^{*}$ atoms as a function of the UV time delay for three different excitation probabilities (blue lines). For comparison, the experimental data are shown as black squares, and the corresponding $\mathrm{MC}$ simulation assuming mobile $\mathrm{He}^{*}$ atoms is shown as a red line. As can be clearly seen, the simulated dynamics for fixed $\mathrm{He}^{*}$ positions proceed on much longer timescales compared to the experimental data, thus showing the critical importance of atomic mobility in the ICD process. To further illustrate this point, Fig. 8(b) shows the relative 

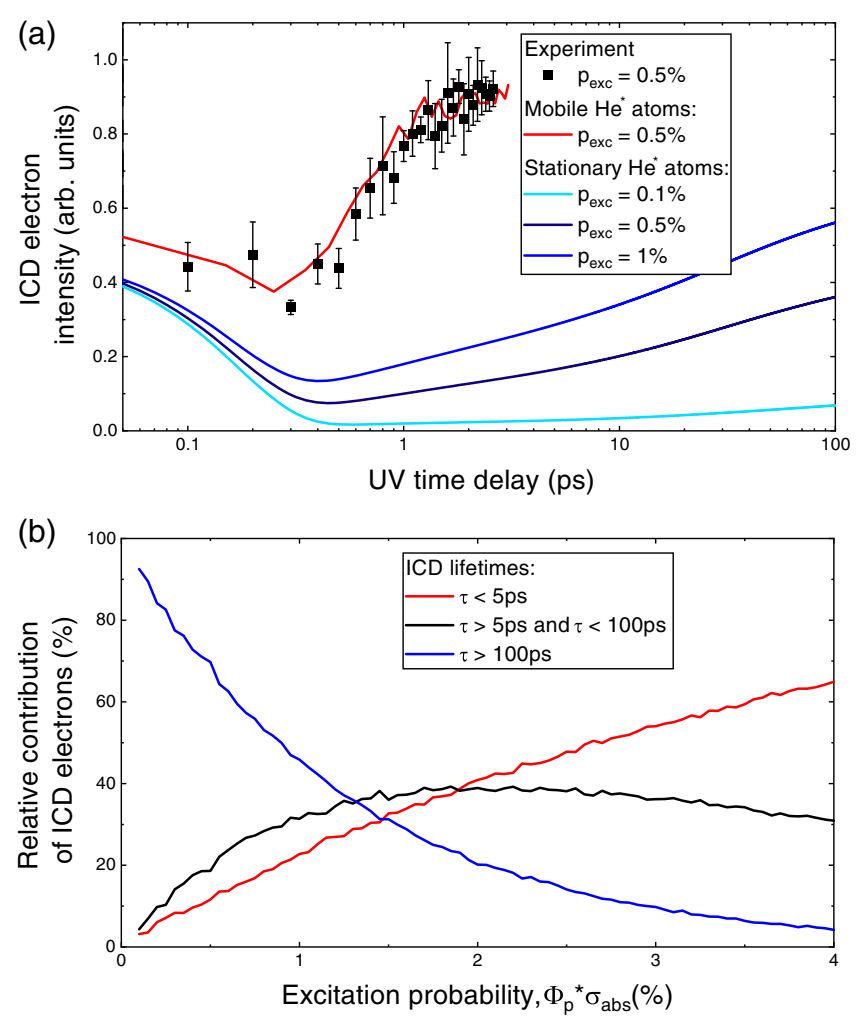

FIG. 8. (a) Simulated ICD electron intensities as a function of the UV time delay for fixed $\mathrm{He}^{*}$ positions with different excitation densities (blue curves). For comparison, the experimental data are shown as black squares, and the corresponding MC simulation assuming mobile $\mathrm{He}^{*}$ atoms is shown as a red line. (b) Relative contribution of ICD electrons broken up into three different ICD lifetime intervals as a function of excitation probability for fixed $\mathrm{He}^{*}$ positions.

contribution of ICD electrons broken up into three different ICD lifetime intervals as a function of excitation probability for fixed $\mathrm{He}^{*}$ positions. For low excitation probability $(\lesssim 1 \%)$, the ICD lifetime would primarily be $\tau>100 \mathrm{ps}$, which is dramatically longer than what is shown in Fig. 2 for similar experimental conditions. Only for high excitation probability $(\gtrsim 2 \%)$ do shorter ICD lifetimes $(\tau<5 \mathrm{ps})$ significantly contribute. We note that at $2.5 \%$ excitation probability the transition from ICD to CAI occurs, in agreement with previous findings [18].

[1] H. Wabnitz, L. Bittner, A. De Castro, R. Döhrmann, P. Gürtler, T. Laarmann, W. Laasch, J. Schulz, A. Swiderski, K. von Haeften et al., Multiple Ionization of Atom Clusters by Intense Soft X-Rays from a Free-Electron Laser, Nature (London) 420, 482 (2002).

[2] C. Bostedt, H. Thomas, M. Hoener, E. Eremina, T. Fennel, K.-H. Meiwes-Broer, H. Wabnitz, M. Kuhlmann, E. Plönjes, $\mathrm{K}$. Tiedtke et al., Multistep Ionization of Argon Clusters in Intense Femtosecond Extreme Ultraviolet Pulses, Phys. Rev. Lett. 100, 133401 (2008).
[3] C. Bostedt, H. Thomas, M. Hoener, T. Möller, U. Saalmann, I. Georgescu, C. Gnodtke, and J.-M. Rost, Fast Electrons from Multi-electron Dynamics in Xenon Clusters Induced by Inner-Shell Ionization, New J. Phys. 12, 083004 (2010).

[4] L. S. Cederbaum, J. Zobeley, and F. Tarantelli, Giant Intermolecular Decay and Fragmentation of Clusters, Phys. Rev. Lett. 79, 4778 (1997).

[5] U. Hergenhahn, Interatomic and Intermolecular Coulombic Decay: The Early Years, J. Electron Spectrosc. Relat. Phenom. 184, 78 (2011).

[6] T. Jahnke, Interatomic and Intermolecular Coulombic Decay: The Coming of Age Story, J. Phys. B 48, 082001 (2015).

[7] T. Jahnke, U. Hergenhahn, B. Winter, R. Dörner, U. Frühling, P. V. Demekhin, K. Gokhberg, L. S. Cederbaum, A. Ehresmann, A. Knie et al., Interatomic and Intermolecular Coulombic Decay, Chem. Rev. 120, 11295 (2020).

[8] K. Gokhberg, P. Kolorenč, A. I. Kuleff, and L. S. Cederbaum, Site- and Energy-Selective Slow-Electron Production through Intermolecular Coulombic Decay, Nature (London) 505, 661 (2014).

[9] F. Trinter, M. Schöffler, H.-K. Kim, F. Sturm, K. Cole, N. Neumann, A. Vredenborg, J. Williams, I. Bocharova, R. Guillemin et al., Resonant Auger Decay Driving Intermolecular Coulombic Decay in Molecular Dimers, Nature (London) 505, 664 (2014).

[10] V. Stumpf, K. Gokhberg, and L. S. Cederbaum, The Role of Metal Ions in X-Ray-Induced Photochemistry, Nat. Chem. 8, 237 (2016).

[11] X. Ren, E. Wang, A. D. Skitnevskaya, A. B. Trofimov, K. Gokhberg, and A. Dorn, Experimental Evidence for Ultrafast Intermolecular Relaxation Processes in Hydrated Biomolecules, Nat. Phys. 14, 1062 (2018).

[12] E. Allaria, R. Appio, L. Badano, W. A. Barletta, S. Bassanese, S. G. Biedron, A. Borga, E. Busetto, D. Castronovo, and P.e. a. Cinquegrana, Highly Coherent and Stable Pulses from the Fermi Seeded Free-Electron Laser in the Extreme Ultraviolet, Nat. Photonics 6, 699 (2012).

[13] E. Allaria, A. Battistoni, F. Bencivenga, R. Borghes, C. Callegari, F. Capotondi, D. Castronovo, P. Cinquegrana, D. Cocco, M. Coreno et al., Tunability Experiments at the FERMI@Elettra Free-Electron Laser, New J. Phys. 14, 113009 (2012).

[14] A. I. Kuleff, K. Gokhberg, S. Kopelke, and L. S. Cederbaum, Ultrafast Interatomic Electronic Decay in Multiply Excited Clusters, Phys. Rev. Lett. 105, 043004 (2010).

[15] A. LaForge, M. Drabbels, N. B. Brauer, M. Coreno, M. Devetta, M. Di Fraia, P. Finetti, C. Grazioli, R. Katzy, V. Lyamayev et al., Collective Autoionization in MultiplyExcited Systems: A Novel Ionization Process Observed in Helium Nanodroplets, Sci. Rep. 4, 3621 (2014).

[16] Y. Ovcharenko, V. Lyamayev, R. Katzy, M. Devetta, A. LaForge, P. O'Keeffe, O. Plekan, P. Finetti, M. Di Fraia, M. Mudrich et al., Novel Collective Autoionization Process Observed in Electron Spectra of He Clusters, Phys. Rev. Lett. 112, 073401 (2014).

[17] D. Iablonskyi, K. Nagaya, H. Fukuzawa, K. Motomura, Y. Kumagai, S. Mondal, T. Tachibana, T. Takanashi, T. Nishiyama, K. Matsunami et al., Slow Interatomic 
Coulombic Decay of Multiply Excited Neon Clusters, Phys. Rev. Lett. 117, 276806 (2016).

[18] Y. Ovcharenko, A. C. LaForge, B. Langbehn, O. Plekan, R. Cucini, P. Finetti, P. O’Keeffe, D. Iablonskyi, T. Nishiyama, K. Ueda, P. Piseri, M. D. Fraia, R. Richter, M. Coreno, C. Callegari, K. C. Prince, F. Stienkemeier, T. Möller, and M. Mudrich, Autoionization Dynamics of Helium Nanodroplets Resonantly Excited by Intense XUV Laser Pulses, New J. Phys. 22, 083043 (2020).

[19] K. Schnorr, A. Senftleben, M. Kurka, A. Rudenko, L. Foucar, G. Schmid, A. Broska, T. Pfeifer, K. Meyer, D. Anielski et al., Time-Resolved Measurement of Interatomic Coulombic Decay in $\mathrm{Ne}_{2}$, Phys. Rev. Lett. 111, 093402 (2013).

[20] T. Takanashi, N. V. Golubev, C. Callegari, H. Fukuzawa, K. Motomura, D. Iablonskyi, Y. Kumagai, S. Mondal, T. Tachibana, K. Nagaya et al., Time-Resolved Measurement of Interatomic Coulombic Decay Induced by Two-Photon Double Excitation of $\mathrm{Ne}_{2}$, Phys. Rev. Lett. 118, 033202 (2017).

[21] J. P. Toennies and A. F. Vilesov, Superfluid Helium Droplets: A Uniquely Cold Nanomatrix for Molecules and Molecular Complexes, Angew. Chem., Int. Ed. 43, 2622 (2004).

[22] M. Shcherbinin, A. C. LaForge, V. Sharma, M. Devetta, R. Richter, R. Moshammer, T. Pfeifer, and M. Mudrich, Interatomic Coulombic Decay in Helium Nanodroplets, Phys. Rev. A 96, 013407 (2017).

[23] F. Wiegandt, F. Trinter, K. Henrichs, D. Metz, M. Pitzer, M. Waitz, E. Jabbour Al Maalouf, C. Janke, J. Rist, N. Wechselberger et al., Direct Observation of Interatomic Coulombic Decay and Subsequent Ion-Atom Scattering in Helium Nanodroplets, Phys. Rev. A 100, 022707 (2019).

[24] D. Buchta, S. R. Krishnan, N. B. Brauer, M. Drabbels, P. O'Keeffe, M. Devetta, M. Di Fraia, C. Callegari, R. Richter, M. Coreno et al., Charge Transfer and Penning Ionization of Dopants in or on Helium Nanodroplets Exposed to EUV Radiation, J. Phys. Chem. A 117, 4394 (2013).

[25] R. Katzy, A. LaForge, Y. Ovcharenko, M. Coreno, M. Devetta, M. Di Fraia, M. Drabbels, P. Finetti, V. Lyamayev, T. Mazza et al., Migration of Surface Excitations in HighlyExcited Nanosystems Probed by Intense Resonant XUV Radiation, J. Phys. B 48, 244011 (2015).

[26] A. C. LaForge, V. Stumpf, K. Gokhberg, J. von Vangerow, F. Stienkemeier, N. V. Kryzhevoi, P. O'Keeffe, A. Ciavardini, S. R. Krishnan, M. Coreno et al., Enhanced Ionization of Embedded Clusters by Electron-Transfer-Mediated Decay in Helium Nanodroplets, Phys. Rev. Lett. 116, 203001 (2016).

[27] A. LaForge, M. Shcherbinin, F. Stienkemeier, R. Richter, R. Moshammer, T. Pfeifer, and M. Mudrich, Highly Efficient Double Ionization of Mixed Alkali Dimers by Intermolecular Coulombic Decay, Nat. Phys. 15, 247 (2019).

[28] L. Ben Ltaief, M. Shcherbinin, S. Mandal, S. Krishnan, A. LaForge, R. Richter, S. Turchini, N. Zema, T. Pfeifer, E. Fasshauer et al., Charge Exchange Dominates Long-Range Interatomic Coulombic Decay of Excited Metal-Doped Helium Nanodroplets, J. Phys. Chem. Lett. 10, 6904 (2019).

[29] L. B. Ltaief, M. Shcherbinin, S. Mandal, S. R. Krishnan, R. Richter, T. Pfeifer, M. Bauer, A. Ghosh, M. Mudrich,
K. Gokhberg et al., Electron Transfer Mediated Decay of Alkali Dimers Attached to He Nanodroplets, Phys. Chem. Chem. Phys. 22, 8557 (2020).

[30] K. von Haeften, T. Laarmann, H. Wabnitz, and T. Möller, Bubble Formation and Decay in ${ }^{3} \mathrm{He}$ and ${ }^{4} \mathrm{He}$ Clusters, Phys. Rev. Lett. 88, 233401 (2002).

[31] M. Mudrich, A. LaForge, A. Ciavardini, P. OKeeffe, C. Callegari, M. Coreno, A. Demidovich, M. Devetta, M. Di Fraia, M. Drabbels et al., Ultrafast Relaxation of Photoexcited Superfluid He Nanodroplets, Nat. Commun. 11, 112 (2020).

[32] H.-B. Zhou, J.-L. Wang, W. Jiang, G.-H. Lu, J. Aguiar, and F. Liu, Electrophobic Interaction Induced Impurity Clustering in Metals, Acta Mater. 119, 1 (2016).

[33] D. Chandler, Interfaces and the Driving Force of Hydrophobic Assembly, Nature (London) 437, 640 (2005).

[34] M. Joppien, R. Karnbach, and T. Möller, Electronic Excitations in Liquid Helium: The Evolution from Small Clusters to Large Droplets, Phys. Rev. Lett. 71, 2654 (1993).

[35] M. Barranco, R. Guardiola, S. Hernández, R. Mayol, J. Navarro, and M. Pi, Helium Nanodroplets: An Overview, J. Low Temp. Phys. 142, 1 (2006).

[36] F. Ancilotto, M. Barranco, F. Coppens, J. Eloranta, N. Halberstadt, A. Hernando, D. Mateo, and M. Pi, Density Functional Theory of Doped Superfluid Liquid Helium and Nanodroplets, Int. Rev. Phys. Chem. 36, 621 (2017).

[37] V. Lyamayev, Y. Ovcharenko, R. Katzy, M. Devetta, L. Bruder, A. LaForge, M. Mudrich, U. Person, F. Stienkemeier, M. Krikunova et al., A Modular End-Station for Atomic, Molecular, and Cluster Science at the Low Density Matter Beamline of FERMI@Elettra, J. Phys. B 46, 164007 (2013).

[38] D. Verma and A. F. Vilesov, Pulsed Helium Droplet Beams, Chem. Phys. Lett. 694, 129 (2018).

[39] B. Dick, Inverting Ion Images without Abel Inversion: Maximum Entropy Reconstruction of Velocity Maps, Phys. Chem. Chem. Phys. 16, 570 (2014).

[40] T. N. Chang and T. K. Fang, Effect of Positive-Energy Orbitals on the Photoionization Cross Sections and Oscillator Strengths of He and Divalent Atoms, Phys. Rev. A 52, 2638 (1995).

[41] See Supplemental Material at http://link.aps.org/ supplemental/10.1103/PhysRevX.11.021011 for additional details on the theoretical calculations and modeling.

[42] T. Miteva, S. Kazandjian, and N. Sisourat, On the Computations of Decay Widths of Fano Resonances, Chem. Phys. 482, 208 (2017).

[43] D. Scharf, J. Jortner, and U. Landman, Vibrational Predissociation Induced by Exciton Trapping in Inert-Gas Clusters, Chem. Phys. Lett. 126, 495 (1986).

[44] A. Scheidemann, B. Schilling, and J. P. Toennies, Anomalies in the Reactions of Helium (1+) with Sulfur Hexafluoride Embedded in Large Helium-4 Clusters, J. Phys. Chem. 97, 2128 (1993).

[45] K. D. Closser, O. Gessner, and M. Head-Gordon, Simulations of the Dissociation of Small Helium Clusters with Ab Initio Molecular Dynamics in Electronically Excited States, J. Chem. Phys. 140, 134306 (2014). 
[46] J. Seong, K. C. Janda, N. Halberstadt, and F. Spiegelmann, Short-Time Charge Motion in $\mathrm{He}_{n}^{+}$Clusters, J. Chem. Phys. 109, 10873 (1998).

[47] A. Hernando, M. Barranco, M. Pi, E. Loginov, M. Langlet, and M. Drabbels, Desorption of Alkali Atoms from ${ }^{4} \mathrm{He}$ Nanodroplets, Phys. Chem. Chem. Phys. 14, 3996 (2012).

[48] H. Buchenau, J. P. Toennies, and J. A. Northby, Excitation and Ionization of ${ }^{4} \mathrm{He}$ Clusters by Electrons, J. Chem. Phys. 95, 8134 (1991).

[49] B. Thaler, S. Ranftl, P. Heim, S. Cesnik, L. Treiber, R. Meyer, A. W. Hauser, W. E. Ernst, and M. Koch, Femtosecond Photoexcitation Dynamics inside a Quantum Solvent, Nat. Commun. 9, 4006 (2018).

[50] K. von Haeften, T. Laarmann, H. Wabnitz, T. Möller, and K. Fink, Size and Isotope Effects of Helium Clusters and Droplets: Identification of Surface and BulkVolume Excitations, J. Phys. Chem. A 115, 7316 (2011).

[51] P. Nijjar, A. Krylov, O. Prezhdo, A. Vilesov, and C. Wittig, Conversion of $\mathrm{He}\left(2^{3} s\right)$ to $\mathrm{He}_{2}\left(a^{3} \sigma_{u}^{+}\right)$in Liquid Helium, J. Phys. Chem. Lett. 9, 6017 (2018).
[52] T. Möller, K. von Haeften, T. Laarman, and R. von Pietrowski, Photochemistry in Rare Gas Clusters, Eur. Phys. J. D 9, 5 (1999).

[53] I. Unger, R. Seidel, S. Thürmer, M. N. Pohl, E. F. Aziz, L. S. Cederbaum, E. Muchová, P. Slavíček, B. Winter, and N. V. Kryzhevoi, Observation of Electron-Transfer-Mediated Decay in Aqueous Solution, Nat. Chem. 9, 708 (2017).

[54] M. Gramlich, B. J. Bohn, Y. Tong, L. Polavarapu, J. Feldmann, and A.S. Urban, Thickness-Dependence of Exciton-Exciton Annihilation in Halide Perovskite Nanoplatelets, J. Phys. Chem. Lett. 11, 5361 (2020).

[55] B. J. Walker, A. J. Musser, D. Beljonne, and R. H. Friend, Singlet Exciton Fission in Solution, Nat. Chem. 5, 1019 (2013).

[56] G. Jones and D. Bradshaw, Resonance Energy Transfer: From Fundamental Theory to Recent Applications, Front. Phys. 7, 100 (2019).

[57] M. P. Ziemkiewicz, D. M. Neumark, and O. Gessner, Ultrafast Electronic Dynamics in Helium Nanodroplets, Int. Rev. Phys. Chem. 34, 239 (2015).

[58] E. Fasshauer and L. B. Madsen, Time-Resolved Spectroscopy of Interparticle Coulombic Decay Processes, Phys. Rev. A 101, 043414 (2020). 